\title{
Objective evaluation of anosmia and ageusia in COVID-19 patients: a single-center experience on 72 cases.
}

\author{
Luigi Angelo Vaira, MD ${ }^{1}$ \\ ${ }^{1}$ University Hospital of Sassari,
}

April 28, 2020

\begin{abstract}
Background. The firsts European case series are detecting a very high frequency of chemosensitive disorders in COVID-19 patients, ranging between $19.4 \%$ and $88 \%$. Methods. Olfactory and gustatory function was objectively tested in 72 COVID19 patients treated at University Hospital of Sassari.Results Overall, 73.6\% of the patients reported having or having had chemosensitive disorders. Olfactory assessment showed variable degree hyposmia in 60 cases and anosmia in 2 patients. Gustatory assessment revealed hypogeusia in 33 cases and complete ageusia in 1 patient. Statistically significant differences in chemosensitive recovery were detected based on age and distance from the onset of clinical manifestations. Conclusion. Olfactory and gustatory dysfunctions represent common clinical findings in COVID-19 patients. Otolaryngologists and head-neck surgeons must by now keep this diagnostic option in mind when evaluating cases of ageusia and nonspecific anosmia that arose suddenly and are not associated with rhinitis symptoms
\end{abstract}

Luigi Angelo Vaira ${ }^{1}$, MD; Giovanna Deiana ${ }^{2}$, MD; Alessandro Giuseppe Fois ${ }^{3}$, MD; Pietro Pirina ${ }^{3}$, MD; Giordano Madeddu ${ }^{4}$, MD; Andrea De Vito ${ }^{4}$, MD; Sergio Babudieri ${ }^{4}, \mathrm{MD}$; Marzia Petrocelli ${ }^{5}$, MD; Antonello Serra $^{6}$, MD; Francesco Bussu ${ }^{7}$, MD; Enrica Ligas ${ }^{1}$ MSN, RN; Giovanni Salzano ${ }^{8}$, MD; Giacomo De Riu ${ }^{1}$ MD, FEBOMFS.

${ }^{1}$ Maxillofacial Surgery Operative Unit, University Hospital of Sassari, Viale San Pietro 43/B, 07100 Sassari, Italy.

${ }^{2}$ Department of Medical, Surgical and Experimental Sciences, University of Sassari, Viale San Pietro, 07100 Sassari, Italy.

${ }^{3}$ Respiratory Diseases Operative Unit, University Hospital of Sassari, Viale San Pietro 43/B, 07100 Sassari, Italy.

${ }^{4}$ Infectious Diseases Operative Unit, University Hospital of Sassari, Viale San Pietro 43/B, 07100 Sassari, Italy.

${ }^{5}$ Maxillofacial Surgery Operative Unit; Bellaria and Maggiore Hospital, AUSL Bologna, Italy.

${ }^{6}$ Surveillance and Prevention Department, University Hospital of Sassari, Via Matteotti 58, 07100 Sassari, Italy.

${ }^{7}$ Otolaryngology Operative Unit, University Hospital of Sassari, Viale San Pietro 43/B, 07100 Sassari, Italy.

${ }^{8}$ Maxillofacial Surgery Unit, University Hospital of Naples "Federico II", Via Pansini 5, 80131 Naples, Italy.

\section{*Corresponding Author:}


Luigi Angelo Vaira

Viale San Pietro 43/B

e-mail: luigi.vaira@gmail.com

tel.: +393401846168

fax: +39079229002

Keywords: COVID-19; anosmia; ageusia; gustatory function; olfactory function.

\section{Abstract}

Background. The firsts European case series are detecting a very high frequency of chemosensitive disorders in COVID-19 patients, ranging between $19.4 \%$ and $88 \%$.

Methods. Olfactory and gustatory function was objectively tested in 72 COVID-19 patients treated at University Hospital of Sassari.

Results Overall, $73.6 \%$ of the patients reported having or having had chemosensitive disorders. Olfactory assessment showed variable degree hyposmia in 60 cases and anosmia in 2 patients. Gustatory assessment revealed hypogeusia in 33 cases and complete ageusia in 1 patient. Statistically significant differences in chemosensitive recovery were detected based on age and distance from the onset of clinical manifestations.

Conclusion. Olfactory and gustatory dysfunctions represent common clinical findings in COVID-19 patients. Otolaryngologists and head-neck surgeons must by now keep this diagnostic option in mind when evaluating cases of ageusia and nonspecific anosmia that arose suddenly and are not associated with rhinitis symptoms

\section{INTRODUCTION}

The rapid spread of severe acute respiratory syndrome coronavirus 2 (SARS-CoV-2) [1] is enabling researchers worldwide to acquire a large amount of clinical data regarding coronavirus disease (COVID-19). The serious health emergency situation makes data collection and analysis very difficult, but some important clinical differences are starting to emerge between Chinese and European case series.

Particularly, smell and taste dysfunctions have been reported as uncommon by Chinese colleagues, during COVID-19 infection. In the only paper present in literature, Mao et al. [2], analyzing neurological manifestation frequency in 214 infected patients, found out anosmia in $11(5.1 \%)$ and ageusia in $12(5.6 \%)$ cases.

In contrast to these data, the firsts European case series [3-6] are detecting a very high frequency of chemosensitive disorders in COVID-19 patients, ranging between 19.4\% and 88\%. In particular, the olfactory and gustative alterations seem especially frequent in the initial stages of the infection and in paucisymptomatic patients [3-5]. These complaints could represent, therefore, pivotal symptoms suspecting SARS-CoV-2 infection [3].

However, all the studies published up to now are anamnestic-observational, presenting the limitation of not reporting any systematic sensitive evaluation of the patients. The objective assessment is fundamental, quantifying the extent of the dysfunction and monitoring the recovery over time.

The purpose of this study is to objectively evaluate the gustatory and olfactory function, through the use of psycho-physiological objective tests, in COVID-19 patients treated in the University Hospital of Sassari.

\section{MATERIALS AND METHODS}

The study was conducted on SARS-CoV-2 positive patients, treated by the COVID departments (Pneumology, Infectious Disease and Surveillance and Prevention Operative Units) of the University Hospital of Sassari, between March 31 and April 6, 2020. 
To be enrolled in the study, patients had to meet the following inclusion criteria: adults over 18 years of age, rhino-pharyngeal swab positive for SARS-CoV-2 infection, patient acceptance for participation in the study.

On the contrary, the study exclusion criteria were: uncooperative patients, assisted ventilation, psychiatric or neurological disorders, previous surgery or radiotherapy in the oral and nasal cavities, pre-existing manifestation of smell and taste alterations, history of head trauma, allergic rhinitis, chronic rhinosinusitis and psychiatric or neurological disorders.

All patients provided informed consent for participation in the study. The study protocol was approved by an independent ethics committee ( $\mathrm{n}^{\circ} 378-2020$-OSS-AUSLBO).

\section{Clinical record assessment}

Some general information were recorded for all the patients: age, gender, praevious clinical history and swab positivity. COVID-19 signs and symptoms were detected from clinical records.

The patients were carefully asked in order to obtain a conceivable timeline of the onset, lasting and the eventual regression, of the chemosensitive symptoms.

\section{Olfactory function assessment}

Both the olfactory threshold and the odor discriminative ability were assessed. All tests were conducted in a quiet room, by the same operators. Olfactory function assessment was carried out by means of the Connecticut Chemosensory Clinical Research Center orthonasal olfaction test (CCCRC) [7-11]. The CCCRC is a simple, validated and widely used test, that includes a butanol threshold assessment and an odor identification test using common odors.

Threshold testing was performed presenting solutions of N-bunatol in deionized waters, decreasing concentration in 8 steps. The strongest butanol concentration was $4 \%$ in $60 \mathrm{~mL}$ of deionized water (bottle 0). Each other bottle (from 1 to 8 ) contained a subsequent 1:3 N-butanol dilution. Two identical squeezable bottles were presented to the patient: one containing the N-butanol solution, Starting from the major dilution, and the other filled with deionized water. The patient was then asked to close one nostril and squeeze the bottle immediately below the other, reporting which of the two bottles smelled most. The threshold was identified when the subject gave the correct answer 4 times. In case of error, the next most concentrated solution was given to the patient. The threshold was quantified for each of the two nostrils with a score from 0 to 8 corresponding to less concentrated bottle that the patient was able to correctly detect. The average between values of the two nostrils expressed the overall score.

For the identification test, common odorants, familiar to the Italian population [Table 1], were placed inside $180 \mathrm{~mL}$ opaque jars covered with gauze. One at a time, the samples were presented to the patient in the same way as the threshold test. Therefore, the patient was asked to identify the odorant on a list containing the 10 test items and 10 distractors [Table 1]. Score ranged from 0 to 10 and was obtained from the average of the two nostrils.

The threshold and identification test scores, after being analyzed separately, were finally converted into the CCCRC composite score. The scoring system is shown in table 2 [Table 2].

\section{Gustatory function assessment}

A standardized and validated test, which investigates the ability to perceive four primary tastes (sweet, salty, sour and bitter) was used to evaluate the gustatory function $[12,13]$. Four solutions, one for each primary taste, were prepared as follows:

- Salted solution: $30 \mathrm{~g}$ of table salt were added to 1 liter of deionized water.

- Sweet solution: $30 \mathrm{~g}$ of refined sugar were dissolved in 1 liter of deionized water.

- Sour solution: $90 \mathrm{~mL}$ of commercial 100\% lemon juice added to 1 liter of deionized water.

- Bitter solution: unsweetened decaffeinated coffee. 
Deionized water was used as control. During the trial, $1 \mathrm{ml}$ of each solution was dropped onto the center of the patient tongue. A different cotton swab was used for each of the solutions and then disposed. The patient was then asked to indicate whether the perceived flavor was sweet, salty, bitter, acid or neutral. The solutions were presented in a random order, excluding the bitter taste, which was always presented last because it tends to alter subsequent taste perception [12].

The answers were recorded as either correct or incorrect. Taste score ranged from 0 to 4 allowing to classify the patients into four categories: Normal (score 4), mild hypogeusia (score 3), moderate hypogeusia (score 2), severe hypogeusia (score 1) and ageusia (score 0).

\section{Statistical analysis}

Statistical analysis was performed using SPSS 26.0 (IBM, Armonk, NY, USA). Categorical variables are reported in numerals and percentages of the total. Descriptive statistics for quantitative variables are given as the mean \pm standard deviation (SD). The statistical analysis of differences in olfactory and gustatory function, between population subgroups, was performed using Student's T test. The level of statistical significance was set at P [?] 0.05 with a $95 \%$ confidence interval.

\section{RESULTS}

Following the inclusion and exclusion criteria, 72 COVID-19 patients (27 males, 45 females, mean age 49.2 years old) were enrolled for the study at the University Hospital of Sassari. Of these, 3 were hospitalized in the Respiratory Diseases Department, 22 in the Infectious Diseases Department, and the remaining 47 patients belonged to the infected healthcare personnel, were quarantined at home and assisted by the Surveillance and Prevention Department. The last group was assessed in occasion of the first recall for the control swab. Table 3 reports a framework summary of patient general characteristics and clinical features [Table 3].

Fifty-three patients (73.6\% of the study population) reported having chemosensory dysfunctions during the course of the COVID-19. Especially, isolated taste disorders were reported in 9 cases (12.5\%), while 14 patients (14.4\%) reported isolated olfactory dysfunctions. Thirty patients (41.7\%) reported they experienced combined disorders of both olfaction and taste. At the time of the evaluation, most of these patients (35 cases, 66\%) reported a complete recovery of chemosensitive functions, which occurred in less than 5 days in 19 cases and in more than 5 days in the remaining 16. On the contrary, 18 patients (34\%) reported a persistence of alterations in taste and olfaction.

\section{Olfactory function assessment results}

Overall, 44 patients (61.1\%) reported having or having had olfactory disorders during SARS-CoV-2 infection. The alterations reported include complete anosmia (34 patients), hyposmia (8 patients) and disosmia (2 patients). Table 4 summarizes the olfactory function assessment results [Table 4]. At the time of the examination, based on the CCCRC scores obtained, complete anosmia was detected in 2 cases $(2.8 \%)$, 58 patients presented variable degree hyposmia (80.6\%) while in 12 cases $(16.7 \%)$ the olfactory function was normal. Twenty-eight patients, who subjectively reported complete olfactory recovery, discordantly presented mild (18 cases) or moderate (10) hyposmia in the CCCRC test.

\section{Gustatory function assessment}

Thirty-nine patients presented, or had presented before the check, taste disorders, subjectively completely resolved in 8 cases. The reported alterations included complete ageusia (28 patients) and variable severity of hypogeusia (11 cases) associated with dysgeusia in 7 patients. Table 5 provides a framework summary of the olfactory function assessment results [Table 5]. Ageusia was detected in 1 patient (1.4\%), severe hypogeusia in 7 patients (9.7\%), moderate hypogeusia in 11 patients (15.3\%) and mild hypogeusia in 16 patients $(22.2 \%)$. Differently, 37 patients $(51.4 \%)$ presented a normal gustatory function by correctly identifying all 4 primary tastes.

\section{Statistical analysis results}


The means of the CCCRC and taste test scores of the different subpopulations studied were analyzed with Student's $\mathrm{T}$ test in order to identify possible correlations between chemosensitive dysfunctions and general or clinical patient characteristics. The results are shown in Table 6 [Table 6]. No statistically significant correlations with the sex of the patients nor with the severity of the pulmonary clinical picture were found. On average, olfactory and gustatory scores were worse in patients over 50 years of age. Anyway, this correlation was statistically significant only for the taste $(\mathrm{P}=0.003)$. Both the olfactory $(\mathrm{P}=0.000)$ and gustatory $(\mathrm{P}$ $=0.001)$ scores showed statistically significant differences between subpopulations selected on the basis of the time elapsed since the onset of symptoms.

\section{DISCUSSION}

After the recent spread of sars-cov-2 in Europe, several authors have reported the high incidence of olfactory and gustatory dysfunctions in COVID-19 patients [3-6]. Particularly, these symptoms appear to be very common in the early stages of the disease [3] and sometimes represent its only clinical manifestation [4]. The identification and isolation of the paucisymptomatic patients is crucial for breaking the virus chain of infection. However, all the reports published so far do not evaluate quantitatively and qualitatively the extent of chemosensory disorders in COVID-19 patients. In most cases the studies are based on subjective selfevaluations of the patients, report of anamnestic data or olfactory and gustatory questionnaire completion, without any objective test or direct medical examination $[4,5,6]$. This can be explained by the emergency situation and the risk of infection approaching these patients without proper personal protection equipment.

This is the first study to objectively evaluate these symptoms. The acquisition of objective data is fundamental to understand mechanisms of olfactory and taste loss in coronavirus infections and might provide new insights into aspects of viral pathogenesis.

The first and most important evidence of the present study is that gustatory and olfactory dysfunctions are very frequent in COVID-19 patients. In our case series, $73.6 \%$ of patients reported having or having had one or both of these problems during the course of the infection. This frequency is much higher than that reported in the anamnestic-observational studies of Vaira et al. [3] and Giacomelli et al. [6], but similar to that found by Lechien et al. [5].

The analysis of the patient's clinical course confirmed that ageusia and anosmia are early symptoms in COVID-19, generally occurring within the firsts 5 days of the clinical onset [Chart 1]. Indeed, in 13 patients of this series (18.1\%), taste and smell impairment represented the first clinical manifestation of the disease. Therefore, otolaryngologists and head-neck surgeons should nowadays contemplate SARS-CoV-2 infection in the differential diagnosis of these non-specific chemosensitive disorders. Sudden onset within 24-48 hours and the absence of nasal obstruction and rhinitic symptoms (in our case series associated in only $9.1 \%$ of cases) are very suspect clinical features of SARS-CoV-2 etiology.

In $66 \%$ of cases, the patient reported spontaneous regression of chemoperceptive dysfunctions at the evaluation time. However, on objective tests, $80 \%$ of these patients still revealed a certain degree of residual hyposmia or hypogeusia. This contradiction could be linked to the presence of a slight previous chronic alteration, in some cases, or to the fact that the patient had noticed such a great improvement, compared to the condition of ageusia and / or anosmia that he had suffered, to consider his current chemopercective capacity as quite normal.

The objective chemosensitive evaluation revealed mostly moderate and mild hyposmia and hypogeusia [Table 4 and 5]. In general, the CCCRC scores were more influenced by a widespread reduction of the olfactory thresholds while the majority of patients, even with moderate and severe hyposmia, maintained a good discriminative ability. Vicks-VapoRoub, which also stimulates trigeminal olfactory sensitivity $[7,8]$, was correctly identified in $97.2 \%$ of cases. Further studies will be needed to clarify the affection of one or both of the olfactory pathways by the virus. As regards the gustatory function, the most affected sensitivities were those for sweet (23 patients) and sour (21 patients), but there was no clear predominance of disturbances towards a primary taste in particular. 
Although most patients reported having suffered complete ageusia and anosmia, these pathological conditions were only detected in a few patients during the tests. Generally, the evaluation was performed at an advanced period of the clinical course (19.3 days from the clinical onset, on average). An earlier evaluation, logistically difficult as the patients in the first days after the diagnosis are uncommonly hospitalized, would be very useful in order to study the olfactory and gustatory functions when they are afflicted the most. In this regard, we are collecting the data of an early evaluation multicenter protocol of COVID-19+ patients quarantined at home, which will be subject to a future publication.

The statistical analysis [Table 6] of the differences in chemosensitive dysfunctions between selected subpopulations, has highlighted some interesting aspects that should be confirmed on larger case studies. Firstly, older patients reported lower CCCRC and taste scores, with a statistically significant difference in taste. Obviously, this data could be influenced by the aging processes that physiologically cause a reduction in chemoperception [14].

The time elapsed since the clinical onset was detect to be the most significantly related factor in taste $(\mathrm{P}=0.001)$ and smell $(\mathrm{P}=0.000)$ alterations. This finding confirmed that the olfactory and gustatory dysfunctions are typical symptoms of the early stages of the infection and over time they tend to disappear or, at least, to decrease. It would also be interesting to monitor these patients over time to establish the long-term evolution of these disorders.

Finally, no significant correlation was detected between the extent of the chemosensitive disorders and the severity of the pulmonary clinical picture. This evidence, which must be confirmed by larger case studies, could open up fascinating pathogenetic scenarios. The pathogenesis of gustatory and olfactory dysfunctions is unclear and only hypotheses can be made based on studies regarding other coronaviruses [15]. The improvement of the chemoceptive function over time, would suggest a competitive action of the virus on the receptors of the olfactory and gustatory cells or local inflammatory phenomena, rather than permanent cell damage. Assuming that, the viral load would certainly play an important role in the pathogenesis of chemosensitive disorders. However, considering that there is a correlation between the severity of the systemic clinical picture and viral load [16], other local factors are likely to partecipate in causing the onset of gustatory and olfactory alterations in COVID-19 patients.

\section{CONCLUSIONS}

Olfactory and gustatory dysfunctions represent common clinical findings in COVID-19 patients. Otolaryngologists and head-neck surgeons must by now keep this diagnostic option in mind when evaluating cases of ageusia and nonspecific anosmia that arose suddenly and are not associated with rhinitis symptoms. The evaluation presented in this study allows to quantify the extent of these disorders by providing an objective and standardized basis that enable to evaluate functional recovery over time. Further studies are needed to investigate more in depth, from a clinical and pathogenetic point of view, chemosensitive disorders in COVID-19 patients.

\section{FUNDING}

None declared

\section{CONFLICT OF INTEREST}

None of the authors has a financial interest in any of the products, devices or drugs mentioned in this manuscript.

ACNOWLEDGEMENT The authors would like to thank all the Italian health workers for the effort and all the sacrifices they are making during this serious health crisis.

\section{REFERENCES}

[1] Wu F, Zhao S, Yu B, Chen YM, Wang W, Song ZG, Hu Y, Tao ZW, Tian JH, Pei YY, Yuan ML, Zhang YL, Dai FH, Liu Y, Wang QM, Zheng JJ, Xu L, Holmes EC, Zhang YZ. A new coronavirus associated with 
human respiratory disease in China. Nature. 2020;579:265-9.

[2] Mao L, Wang M, Chen S. He Q. Chang J, Hong C, Zhou Y, Wang D, Yanan Li, Huijuan J, Hu B. Neurological manifestations of hospitalized patients with COVID-19 in Wuhan, China: a retrospective case series study. MedRXiv.https://doi.org/10.1101/2020.02.22.200.26500.

[3] Vaira LA, Salzano G, Deiana G, De Riu G. Anosmia and ageusia: common findings in COVID-19 patients. Laryngoscope. 2020. Doi: 10.1002/lary.28692.

[4] Gane SB, Kelly C, Hopkins C. Isolated sudden onset anosmia in COVID-19 infection. A novel syndrome? Rhinology. 2020. Doi: 10.4193/Rhin20.114.

[5] Lechien JR, Chiesta-Estomba CM, De Siati DR, Horoi M, Le Bon SD, Rodriguez A, Dequanter A, Dequanter D, Blecic S, El Afia F, Distinguin L, Chekkoury-Idrissi Y, Hans S, Delgado IL, Calvo-Henriquez C, Lavigne P, Falanga C, Barillari MR, Cammaroto G, Khalife M, Leich P, Souchay C, Rossi C, Journe F, Hsieh J, Edjlali M, Carlier R, RIs L, Lovato A, De Filippis C, Coppee F, Fakhry N, Ayad T, Saussez S. Olfactory and gustatory dysfunctions as a clinical presentation of mild-to-moderate forms of the coronavirus disease (COVID-19): a multicenter European study.

[6] Giacomelli A, Pezzati L, Conti F, Bernacchia D, Siano M, Orelni L, Rusconi S, Gervasoni C, Ridolfo AL, Rizzardini G, Antinori S, Galli M. Self-reported olfactory and taste disorders in SARS-CoV-2 patients: a cross-sectional study. Clin Infect Dis. 2020 Doi: 10.1093/cid/ciaa330.

[7] Cain WS, Gent, J, Catalanotto FA, Goodspeed RB. Clinical evaluation of olfaction. Am J Otolaryngol. 1983;4:252-6.

[8] Cain WS, Gent JF, Goodspeed RB, Leonard G. Evaluation of olfactory dysfunction in the Connecticut Chemosensory Clinical Research Center. Laryngoscope. 1988;98:83-8.

[9] Leon EA, Catalanotto FA, Werning JW. Retronasal and orthonasal olfactory ability after laryngectomy. Arch Otolaryngol Head Neck Surg. 2007;133:32-6.

[10] Aksoy C, Elsurer C, Artac H, Bozkurt MK. Evaluation of olfactory function in children with seasonal allergic rhinitis and its correlation with acoustic rhinometry. Int J Ped Otorhinolaryngol. 2018;113:188-91.

[11] Veyseller B, Ozucer B, Karaaltin AB, Yildirim Y, Degirmenci N, Aksoy F, Ozturan O. Connecticut (CCCRC) olfactory test: normative values in 426 healthy volunteers. Indian J Otolaryngol Head Neck Surg. 2014;66:31-4.

[12] Massarelli O, Vaira LA, Biglio A, Gobbi R, Dell'aversana Orabona G, De Riu G. Sensory recovery of myomucosal flap oral cavity reconstructions. Head Neck. 2018;40:467-74.

[13] Loewen IJ, Boliek CA, Harris J, Seikaly H, Rieger JM. Oral sensation and function: a comparison of patients with innervated radial forearm free flap reconstruction to healthy matched controls. Head Neck. 2010;32:85-95

[14] Doty RL. Age-related deficits in taste and smell. Otolaryngol Clin North Am. 2018;51:815-25.

[15] Vaira LA, Salzano G, Piombino P, De Riu G. Pathogenesis of ageusia and anosmia in COVID-19 patients: a literature review. 2020 (Manuscript submitted for publication)

[16] Liu Y, Yang Y, Zhang C, Huang F, Wang D, Yuan J, Wang Z, Li J, Li J, Feng C, Zhang Z, Wang L, Peng L, Chen L, Quin Y, Zhao D, Tan S, Yin L, Xu J, Zhou C, Jiang C, Liu L. Clinical and biochemical indexes from 2019-nCoV infected patients linked to viral loads and lung injury. Sci China Life Sci. 2020;63:364-74.

TABLE LEGEND

\section{Hosted file}


HED-20-0524_Table_1.docx available at https://authorea.com/users/312328/articles/443262-objectiveevaluation-of-anosmia-and-ageusia-in-covid-19-patients-a-single-center-experience-on-72-cases

Table 1. Identification test 20 item list $[7,8]$

\section{Hosted file}

HED-20-0524_Table_2.docx available at https://authorea.com/users/312328/articles/443262-objectiveevaluation-of-anosmia-and-ageusia-in-covid-19-patients-a-single-center-experience-on-72-cases

Table 2. Connecticut Chemosensory Clinical Research Center (CCCRC) test composite scoring system [7].

\section{Hosted file}

HED-20-0524_Table_3.docx available at https://authorea.com/users/312328/articles/443262-objectiveevaluation-of-anosmia-and-ageusia-in-covid-19-patients-a-single-center-experience-on-72-cases

Table 3. General and clinical characteristics of the study population

\section{Hosted file}

HED-20-0524_Table_4 (1).docx available at https://authorea.com/users/312328/articles/443262objective-evaluation-of-anosmia-and-ageusia-in-covid-19-patients-a-single-center-experienceon-72-cases

Table 4. Connecticut Chemosensory Clinical Research Center (CCCRC) test results

\section{Hosted file}

HED-20-0524_Table_5.docx available at https://authorea.com/users/312328/articles/443262-objectiveevaluation-of-anosmia-and-ageusia-in-covid-19-patients-a-single-center-experience-on-72-cases

Table 5. Gustatory function assessment results

\section{Hosted file}

HED-20-0524_Table_6.docx available at https ://authorea.com/users/312328/articles/443262-objectiveevaluation-of-anosmia-and-ageusia-in-covid-19-patients-a-single-center-experience-on-72-cases

Table 6. Statistical analysis results

\section{CHART LEGEND}

\section{Hosted file}

HED-20-0524_Chart_1.jpg available at https : //authorea.com/users/312328/articles/443262-objectiveevaluation-of-anosmia-and-ageusia-in-covid-19-patients-a-single-center-experience-on-72-cases Chart 1 . Olfactory and gustatory dysfunction clinical course. 